\title{
Nuclear-spin effects in singly negatively charged InP quantum dots
}

\author{
Bipul Pal, ${ }^{1, *}$ Sergey Yu. Verbin,,${ }^{2,3,}$ Ivan V. Ignatiev, ${ }^{2,3}$ Michio Ikezawa, ${ }^{1}$ and Yasuaki Masumoto ${ }^{1}$ \\ ${ }^{1}$ Institute of Physics and Tsukuba Advanced Research Alliance (TARA), University of Tsukuba, Tsukuba 305-8571, Japan \\ ${ }^{2}$ Institute of Physics, St. Petersburg State University, St. Petersburg 198504, Russia \\ ${ }^{3}$ Venture Business Laboratory, University of Tsukuba, Tsukuba 305-8571, Japan
}

(Received 21 November 2006; revised manuscript received 24 January 2007; published 26 March 2007)

\begin{abstract}
Experimental investigation of nuclear-spin effects on the electron-spin polarization in singly negatively charged InP quantum dots is reported. Pump-probe photoluminescence measurements of electron-spin relaxation in the microsecond time scale are used to estimate the time period $T_{N}$ of the Larmor precession of nuclear spins in the hyperfine field of electrons. We find $T_{N}$ to be $\sim 1 \mu$ s at $T \approx 5 \mathrm{~K}$, under the vanishing external magnetic field. From the time-integrated measurements of electron-spin polarization as a function of a longitudinally applied magnetic field at $T \approx 5 \mathrm{~K}$, we find that the Overhauser field appearing due to the dynamic nuclear polarization increases linearly with the excitation power, though its magnitude remains smaller than $10 \mathrm{mT}$ up to the highest excitation power $(50 \mathrm{~mW})$ used in these experiments. The effective magnetic field of the frozen fluctuations of nuclear spins is found to be $15 \mathrm{mT}$, independent of the excitation power.
\end{abstract}

DOI: 10.1103/PhysRevB.75.125322

\section{INTRODUCTION}

Strong localization of electrons in quantum dots (QDs) may enhance the hyperfine interaction of electron spins with those of nuclei. ${ }^{1}$ Various aspects of the hyperfine interaction of electron and nuclear spins have been studied for last three decades in different materials, ${ }^{2}$ including InP QDs. ${ }^{3,4}$ Charge-tunable InP QDs with one resident electron per dot, on an average, have recently attracted considerable research interest due to the observation of submillisecond spin lifetime of resident electrons in these QDs. ${ }^{5,6}$ This observation makes it a promising candidate for quantum memory element in the emerging fields of quantum information technology and spintronics. ${ }^{7}$ Application of QDs as the building blocks of quantum computers has been proposed. ${ }^{8}$

However, the influence of the hyperfine interaction between electron and nuclear spins on the long-lived electronspin polarization needs to be clarified. Two effects of the electron-nuclear spin-spin interactions may be considered. One of them is the so-called dynamic nuclear polarization. ${ }^{2}$ In the optical orientation of electron spins in semiconductors by circularly polarized photons, the spin-oriented electrons dynamically polarize the nuclear spins due to the hyperfine coupling of the electron- and nuclear-spin subsystems. ${ }^{2,9}$ In turn, the spin-polarized nuclei produce an effective internal magnetic field (the Overhauser field $B_{N}$ ), which may influence the electron-spin dynamics. In the presence of an external magnetic field $B_{\text {ext }}$, electron spins should feel a total magnetic field $B_{T}=B_{\text {ext }}+B_{N}$.

Another important effect of the electron-nuclear hyperfine coupling is the electron-spin relaxation via its interaction with nuclear spins. ${ }^{10-12}$ At low temperature, relaxation of the coupled electron-nuclear spin system is determined by three processes, namely, (i) electron-spin precession in the frozen fluctuations of the hyperfine field of the nuclear spins, (ii) nuclear-spin precession in the hyperfine field of the electron spins, and (iii) nuclear-spin relaxation in the dipole-dipole field of its nuclear neighbors. These three processes have very different characteristic time scales. Theoretical estimate for GaAs QDs containing $\sim 10^{5}$ nuclei suggests them to be $\sim 1 \mathrm{~ns}, \sim 1 \mu \mathrm{s}$, and $\sim 100 \mu \mathrm{s}$, respectively. ${ }^{10}$ The last one of
PACS number(s): 78.67.Hc, 71.35.Pq, 72.25.Fe, 72.25.Rb

the above processes will not be considered further in this paper, because it affects the electron-spin dynamics over a long time scale of $10^{-4}-10^{-3} \mathrm{~s}$. In this long time regime, many other mechanisms, such as those originating from the spin-orbit coupling and interaction with phonons, become important for electron spin-relaxation.

The first relaxation process mentioned above arises from the fact that due to a large but limited number of nuclear spins, typically $n \sim 10^{5}$, interacting with the electron spin in a QD, random correlation of nuclear spins may create a fluctuating nuclear polarization, $\Delta F_{N} \propto F_{N} / \sqrt{n}$, where $F_{N}$ is the total spin of the polarized nuclei. Fluctuation $\Delta F_{N}$ acts on the electron spin as another internal magnetic field $B_{f}$, with random magnitude and orientation over the QD ensemble. ${ }^{10} \mathrm{We}$ may note that the Larmor precession of the nuclear spins in the hyperfine field of an electron spin is much slower than that of the electron spin in the nuclear hyperfine field, because the interaction of an electron spin with a single nucleus is $\sqrt{n}$ times weaker compared to its interaction with the effective magnetic field of the nuclear fluctuations. Thus, the electron "sees" a snapshot of the "frozen fluctuations" of the nuclear field. Electron-spin precession in the magnetic field $B_{f}$ is expected to cause electron spin relaxation in the QD ensemble in a time scale of the order of $1 \mathrm{~ns}$, during which the electron-spin polarization decays to one-third of its initial value. After the initial decay in the nanosecond time scale, the remaining spin polarization relaxes very slowly over a time scale much longer than the radiative lifetime of the electron-hole pair. ${ }^{10}$

Electron-spin relaxation due to the frozen fluctuations of nuclear spins (FFNS) may be suppressed by a longitudinally (along the optical excitation axis) applied magnetic field with a magnitude larger than $B_{f} .{ }^{10}$ Recent study of electron-spin relaxation in $p$-doped InAs QDs by Braun et al. ${ }^{13}$ found that at zero external magnetic field, electron-spin polarization decays down to one-third of its initial value within 800 ps after photoexcitation. Then, the residual spin polarization remains stable with no measurable decay within the photoluminescence (PL) decay time. The authors found that the initial fast relaxation of electron spin was suppressed by the application 
of a small $(\sim 100 \mathrm{mT})$ external magnetic field. We want to note the fact that for $B_{\mathrm{ext}}=0$, two distinctly different time regimes are present in the electron-spin relaxation. ${ }^{14}$ The initial fast relaxation is caused by the random distribution of $B_{f}$ in the QD ensemble. ${ }^{10,13}$ However, a total depolarization of electron spins does not take place in this regime. The residual electron-spin polarization decays very slowly in a second relaxation regime, which is governed by the slow timevarying change in the distribution of $B_{f}$. This slow change in the distribution of $B_{f}$ is caused by the variation in the precession rate of nuclear spins in the hyperfine field of electrons. ${ }^{10,13}$ We denote it as the nuclear-spin precession effect (NSPE). Study of electron-spin relaxation due to the NSPE in the microsecond time range should give an estimate of the nuclear-spin precession period $T_{N}$ in the hyperfine field of electrons. However, to the best of our knowledge, this has not been experimentally studied so far.

In this paper, we describe our experimental study of nuclear-spin effects on the long-lived spin polarization of resident electrons, observed recently ${ }^{5,6}$ in the singly negatively charged InP QDs. Electron-spin dynamics and the influence of nuclear spins on it are probed by the time-resolved as well as time-integrated measurements of the degree of PL circular polarization $\rho_{c}$, defined quantitatively in Sec. II. Our time-domain measurements of $\rho_{c}$ by using a PL pump-probe technique $e^{5,6,15,16}$ in the microsecond time regime reveal electron-spin relaxation via the NSPE at the vanishing external magnetic field. From the value of the electron-spin decay time $\tau_{d}$ for $B_{\text {ext }}=0$, we estimate that $T_{N} \sim 1 \mu \mathrm{s}$ at $T \approx 5 \mathrm{~K}$, comparable to the theoretical estimate available for GaAs QDs. ${ }^{10}$ We also measure the dependence of $\rho_{c}$ on $B_{\text {ext }}$ in time-integrated measurements at $T \approx 5 \mathrm{~K}$. From these steadystate measurements, we obtain the Overhauser field $B_{N}=6 \mathrm{mT}$ at $50 \mathrm{~mW} \mathrm{cw}$ excitation and the effective magnetic field of the FFNS, $B_{f}=15 \mathrm{mT}$, independent of the excitation power. The relatively small value of $B_{N}$ may come from efficient leakage of QD nuclear-spin polarization to the surrounding lattice nuclei.

\section{EXPERIMENT}

Our sample consists of a single layer of self-assembled InP QDs, embedded between $\mathrm{Ga}_{0.5} \mathrm{In}_{0.5} \mathrm{P}$ barriers grown on a $n^{+}-$GaAs substrate. The average base diameter (height) of the QDs is $\sim 40(5) \mathrm{nm}$ and the areal density of dots is $\sim 10^{10} \mathrm{~cm}^{-2}$. Semitransparent indium tin oxide electrode is deposited on top of the sample to control the charge state of the QDs by means of an applied electric bias. ${ }^{5,6,17}$ For the present study on the singly negatively charged QDs, we apply an electric bias of $U_{b}=-0.1 \mathrm{~V}$. This is because it was found from a previous study of trionic quantum beats ${ }^{17}$ on the same sample that at $U_{b} \approx-0.1 \mathrm{~V}$, the QDs contain one resident electron per dot, on an average.

Electron spins in the QD ensemble are polarized in our experiments by using the well-known optical orientation technique. ${ }^{2,18}$ We excite the QDs quasiresonantly (in the excited state of the QDs, but below the wetting layer band gap) by a circularly polarized beam from a Ti:sapphire laser, which can be operated either in $\mathrm{cw}$ or in pulsed mode. The

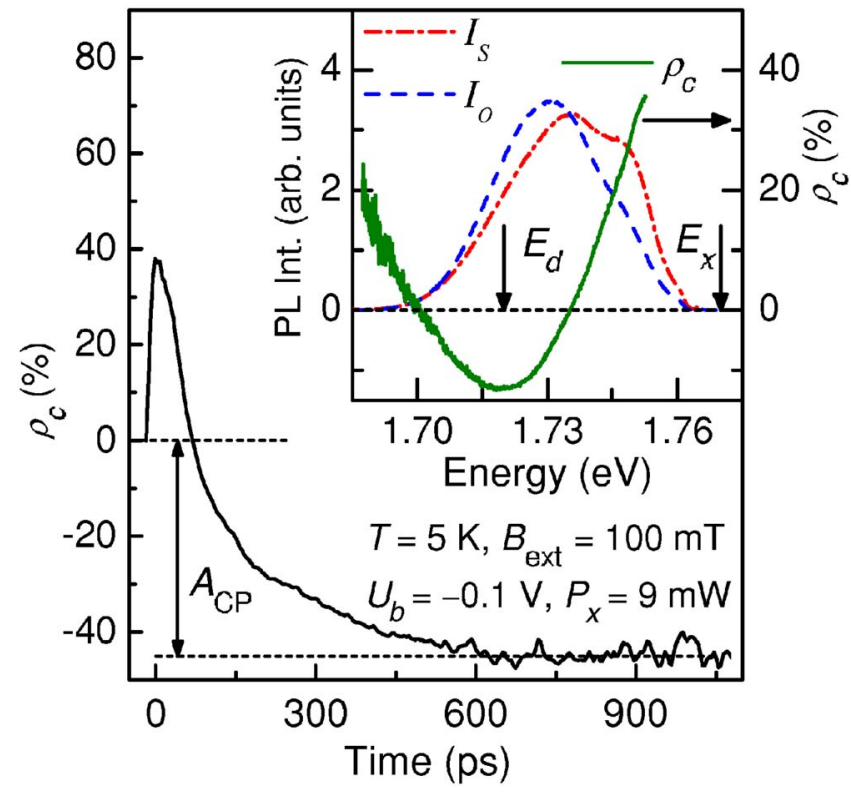

FIG. 1. (Color online) Time dependence of $\rho_{c}$ measured for $E_{x}$ and $E_{d}$ indicated in the inset. $B_{\text {ext }}=100 \mathrm{mT}$ was applied to suppress the effect of the FFNS. The inset shows the spectra of $I_{S}$ and $I_{O}$. Spectral dependence of $\rho_{c}$ is shown for $E_{x}$ indicated by an arrow.

excitation beam is directed along the sample growth axis and is focused to a spot size of $\sim 150 \mu \mathrm{m}$ in diameter on the sample kept in a magneto-optical cryostat at $T \approx 5 \mathrm{~K}$. The excitation energy $E_{x}=1.77 \mathrm{eV}$ and the detection energy $E_{d}=1.72 \mathrm{eV}$ used in our experiments are indicated by arrows on the polarization-resolved PL spectra in Fig. 1 (inset). These spectra are measured by using suitable combinations of retardation plates and Glan-Thompson linear polarizers and a triple spectrometer (focal length of $1 \mathrm{~m}$ ) equipped with a charge-coupled device detector. The spectral resolution is $0.05 \mathrm{meV}$. We monitor the degree of circular polarization $\rho_{c}=\left(I_{S}-I_{O}\right) /\left(I_{S}+I_{O}\right)$ for the ground-state PL. Here $I_{S}\left(I_{O}\right)$ is the intensity of the PL component having the same (opposite) circular polarization as that of the excitation beam. We study $\rho_{c}$, in the time-integrated as well as time-resolved measurements, as a function of the external magnetic field $B_{\text {ext }}$ applied along the optical excitation axis (longitudinal magnetic field, Faraday geometry). Time-resolved data are taken by using a synchroscan streak camera, while for the timeintegrated measurements, a GaAs photomultiplier tube and a two channel gated photon counter are used.

\section{RESULTS AND DISCUSSION}

\section{A. Negative circularly polarized PL}

Our measurements of polarization-resolved PL spectra under quasiresonant excitation $\left(E_{x}=1.77 \mathrm{eV}\right)$ of singly negatively charged InP QDs show that the degree of circular polarization $\rho_{c}$ is negative ${ }^{5,6,19,20}$ in the spectral region $1.7-1.735 \mathrm{eV}$, for which $\Delta E=\left(E_{x}-E_{d}\right)$ lies between 70 and $35 \mathrm{meV}$ [Fig. 1 (inset)]. We measure the time dependence of $\rho_{c}$ for $E_{d}=1.72 \mathrm{eV}$. The data are plotted in Fig. 1. Initially, $\rho_{c}$ is seen to be positive, but it becomes negative at 70 ps after 


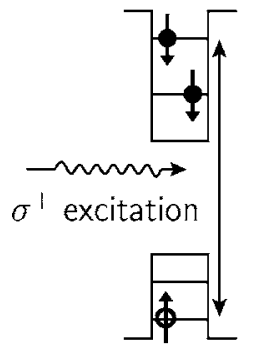

(i)

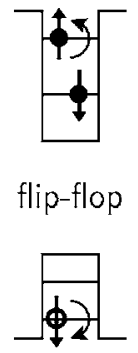

(ii)

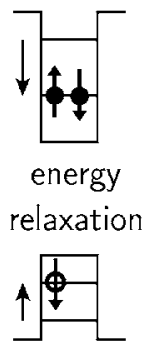

(iii)

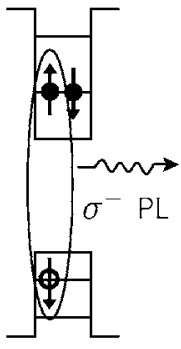

(iv)
FIG. 2. A schematic model to explain the occurrence of the negative value of $\rho_{c}$ due to the presence of the optically oriented resident electron spins in the QDs.

the excitation pulse and then $\rho_{c}$ approaches a constant negative value. We denote this constant value as the amplitude of circular polarization of PL $\left(A_{\mathrm{CP}}\right)$ (see Fig. 1). The value of $A_{\mathrm{CP}}$ increases logarithmically with the excitation power ${ }^{24}$ and reaches up to $45 \%$. In the time-integrated measurements, negative value of $\rho_{c}$ reaches up to $30 \%$.

The remarkable negative circular polarization of $P L$ is related to the optically created spin orientation of the resident electrons. How the sign of $\rho_{c}$ is determined by the spin direction of the resident electron in a $\mathrm{QD}$ is discussed in many papers, see, e.g., Refs. 5, 6, 15, 21, 25, and 26. Here, we briefly explain how the negative degree of circular polarization of PL arises for our experimental condition due to the presence of the optically polarized resident electron spins in the QDs. For this, we refer to the schematic diagram in Fig. 2. Quasiresonant excitation in our experiments creates electron-hole pair in the QD excited state. As the resident electron spins in the QDs are polarized by the excitation photons, ${ }^{18}$ the spin of the photogenerated electron in the excited state and that of the resident electron in the ground state should have a parallel orientation in the majority of the QDs in the ensemble. For simplicity, we consider here only these QDs [Fig. 2(i)], as $\rho_{c}$ for the QD ensemble is mainly determined by them. In these QDs, a direct energy relaxation of the photogenerated electron to the ground state is blocked due to Pauli exclusion principle. However, the electron-hole pair in the excited state can undergo a flip-flop transition, ${ }^{6,21,22}$ in which a simultaneous flip of the electron and hole spins takes place [Fig. 2(ii)]. This is followed by energy relaxation of both the hot carriers [Fig. 2(iii)]. The flip-flop transition is caused by the anisotropic exchange interaction in QDs. ${ }^{21,25,26}$ After the flip-flop transition, PL emitted from the radiative recombination of the spin-flipped electron and hole in the ground state has the opposite circular polarization compared to the excitation photons [Fig. 2(iv)]. Thus, the degree of PL circular polarization becomes negative. We assume that the hole spin relaxation time in the QD ground state is much longer than the radiative lifetime. ${ }^{27,28}$ This is supported by the data in Fig. 1, where $\rho_{c}$ approaches a constant negative value and remains stable over the PL decay time.

The measurements of $\rho_{c}$ in the time-integrated experiments and of $A_{\mathrm{CP}}$ in the time-resolved experiments are used in this work for the study of nuclear-spin effects on the spin polarization of resident electrons in the singly negatively charged QDs.

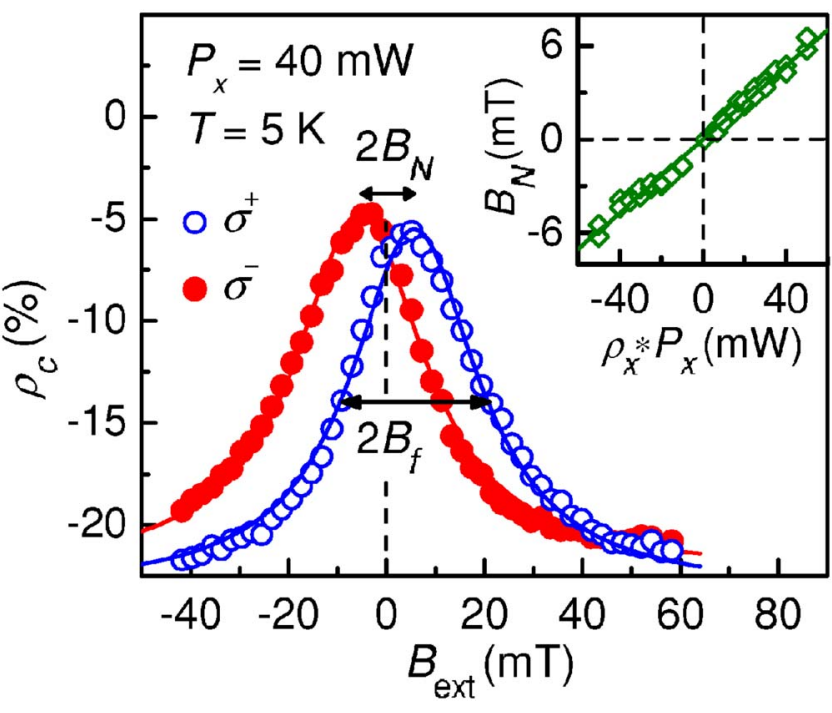

FIG. 3. (Color online) Dependence of $\rho_{c}$ on $B_{\text {ext }}$ for $\sigma^{+}$- and $\sigma^{-}$-polarized cw excitations. The solid lines are Lorentzian fits used to estimate $B_{f}$. Shifts of the minima of $\left|\rho_{c}\right|$ from $B_{\text {ext }}=0$ estimate $B_{N}$. The inset shows the linear dependence of $B_{N}$ on the scaled excitation power $\rho_{x} * P_{x}$, where $\rho_{x}=+1(-1)$ for $\sigma^{+}\left(\sigma^{-}\right)$excitation and $P_{x}$ is excitation power.

\section{B. Frozen fluctuations of nuclear hyperfine field}

It turns out that the PL circular polarization, and hence, the electron-spin polarization, is very sensitive to $B_{\text {ext }}$. Time-integrated measurements in Fig. 3 show the dependence of $\rho_{c}$ on $B_{\text {ext }}$ for $\sigma^{+}$- and $\sigma^{-}$-polarized cw excitations. As seen there, $\rho_{c}$ becomes nearly independent of $B_{\text {ext }}$ for $B_{\text {ext }}>50 \mathrm{mT}$. Absolute value of $\rho_{c}$ decreases with decreasing $\left|B_{\text {ext }}\right|$ and reaches a minimum for $\left|B_{\text {ext }}\right|$ nearly, but not exactly, zero. The behavior of $\rho_{c}$ as a function of $B_{\text {ext }}$ can be fitted well by a Lorentzian with a half-width at half maximum of $15 \mathrm{mT}$.

The decrease of $\left|\rho_{c}\right|$ may be interpreted as the effect of electron-spin relaxation in the QD ensemble by the field $B_{f}$ of the FFNS. ${ }^{10}$ The effect is suppressed by the external magnetic field when $B_{\text {ext }}$ exceeds $B_{f}$ in magnitude, allowing electron-spin polarization and, hence, $\left|\rho_{c}\right|$ to increase to reach a steady-state value. So, the value of $B_{f}$ may be estimated from the half-width at half maximum of the Lorentzians in Fig. 3. Thus, we estimate a value of $B_{f}=15 \mathrm{mT}$ for the QDs under study. The obtained value of $B_{f}$ is found to be independent of the excitation power up to the highest excitation power $(50 \mathrm{~mW})$ used, suggesting that it is intrinsic to the InP QDs. For $B_{f}=15 \mathrm{mT}$, we estimate the electron-spin relaxation time $\tau_{s}=\hbar /\left(g_{e} \mu_{B} B_{f}\right) \approx 0.5 \mathrm{~ns}\left[\mu_{B}=\right.$ Bohr magneton and $g_{e}=$ electron Landé $g$ factor $=1.5$ (Ref. 29)], resulting from the FFNS. The values of $B_{f}=15 \mathrm{mT}$ and $\tau_{s}=0.5 \mathrm{~ns}$ obtained here for the InP QDs are comparable to those theoretically estimated by Merkulov et al. ${ }^{10}$ for GaAs QDs and to those experimentally obtained by Braun et al. ${ }^{13}$ for InAs QDs.

\section{Dynamic nuclear polarization}

As seen in Fig. 3, the minima of $\left|\rho_{c}\right|$ are shifted from $B_{\text {ext }}=0$. This is caused by the Overhauser field $B_{N}$, due to 
which the electron spin "feels" a total magnetic field $B_{T}=B_{N}+B_{\text {ext }}$ in the presence of the external magnetic field $B_{\text {ext }}$. When averaged over the QD ensemble, the field $B_{f}$ of the FFNS does not contribute to the total magnetic field. However, it causes electron-spin relaxation and, hence, decrease in $\left|\rho_{c}\right|$ when $B_{T}$ approaches zero. Thus, the minimum of $\left|\rho_{c}\right|$ should be observed at $B_{\text {ext }}=-B_{N}$. This allows us to estimate $B_{N}$. The sign of $B_{N}$ created by light should be opposite for the $\sigma^{+}$- and $\sigma^{-}$-polarized excitations. As a result, the minima of $\left|\rho_{c}\right|$ for $\sigma^{+}$- and $\sigma^{-}$-polarized excitations are shifted symmetrically from $B_{\text {ext }}=0$ in opposite directions in Fig. 3. ${ }^{30}$ The minima in the two cases differ by $2 B_{N}$. We study the excitation power $\left(P_{x}\right)$ dependence of $B_{N}$. A plot of $B_{N}$ as a function of the scaled excitation power $\rho_{x} * P_{x}$, where the helicity $\rho_{x}=+1(-1)$ for $\sigma^{+}\left(\sigma^{-}\right)$excitation, shows that the dynamic nuclear polarization builds up linearly with the excitation laser power [Fig. 3 (inset)].

We find experimentally that up to $P_{x}=50 \mathrm{~mW}, B_{N}$ remains smaller than $10 \mathrm{mT}$ and that $B_{N}$ does not show any indication of saturation. The value of $B_{N}=6 \mathrm{mT}$ observed at $P_{x}=50 \mathrm{~mW}$ in our experiments is in agreement with previous reports of $B_{N}$ measured in an ensemble of InP nanoislands embedded in $\mathrm{InGaP}$ matrix. ${ }^{3} \mathrm{~A}$ value of $B_{N}=1.2 \mathrm{~T}$ for GaAs QDs formed by interface nanoroughness in a GaAs quantum well has been reported by Gammon et al. ${ }^{1}$ and Brown et al. $^{31}$ in single dot measurements, where they estimated that $65 \%$ of the nuclear spins were polarized. Yokoi et $a l .{ }^{32}$ have reported $B_{N}=160 \mathrm{mT}$ by using single dot spectroscopy for self-assembled InAlAs QDs, where 6\% of the nuclei were polarized. The origin of the small $B_{N}$ observed for self-assembled InP QD ensemble is not fully clear. We may say that only a small fraction of the nuclei in a QD are polarized, because we do not observe any saturation of $B_{N}$ up to the highest excitation power used. This may be caused by the low excitation efficiency of the QDs under quasiresonant excitation we used and also by the inefficient transfer of spin polarization from electrons to nuclei. ${ }^{33}$ Another possible reason may be the efficient leakage of nuclear-spin polarization from the QDs to the surrounding lattice nuclei. ${ }^{2}$ Due to the large nuclear spin of In $(I=9 / 2)$, it may lose its spin polarization rather efficiently through quadrupole interaction in the presence of a time-varying gradient of local electric field, which may be created by the photogenerated electrons in the QDs. ${ }^{34}$ Then, due to close proximity, spin polarization of $\mathrm{P}$ nuclei $(I=1 / 2)$ may be transferred to In nuclei and eventually lost to the surrounding lattice nuclei due to efficient spin relaxation of In nuclei.

\section{Electron-spin relaxation by slow variation in frozen fluctuations of nuclear spins}

For a direct time-domain study of electron-spin relaxation, we use a pump-probe PL technique. .,6,15,16 One of the main advantage of this technique is that the measurable time range of the spin dynamics by this method is not limited by the PL lifetime, in contrast to the time-resolved PL measurements, where spin relaxation can be monitored only within the PL decay time. ${ }^{13}$ Details of our pump-probe PL experimental arrangement are discussed in Refs. 5 and 6. In this

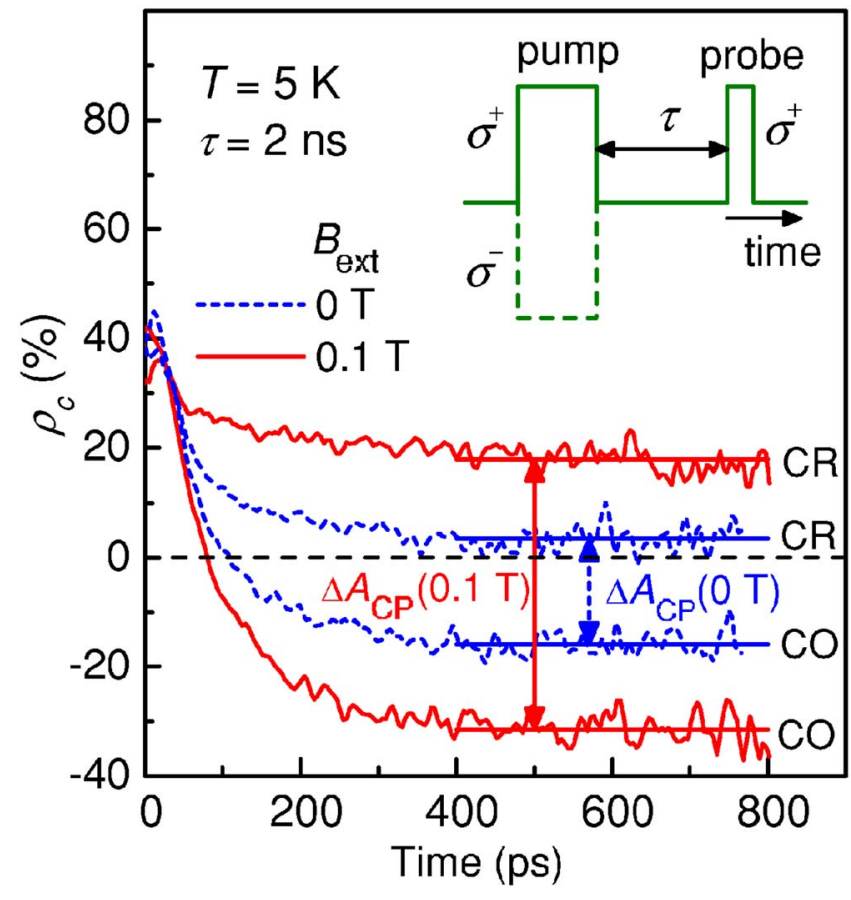

FIG. 4. (Color online) Kinetics of $\rho_{c}$ for the probe PL at $\tau=2 \mathrm{~ns}$ for $B_{\mathrm{ext}}=0$ and $0.1 \mathrm{~T}$. The pump and probe beams are either cocircularly $(\mathrm{CO})$ or cross-circularly $(\mathrm{CR})$ polarized. The difference $\Delta A_{\mathrm{CP}}$ between $A_{\mathrm{CP}}$ for the $\mathrm{CR}$ and $\mathrm{CO}$ cases is indicated by arrows for $B_{\text {ext }}=0$ and $0.1 \mathrm{~T}$. It is found that $\Delta A_{\mathrm{CP}}(0 \mathrm{~T}) / \Delta A_{\mathrm{CP}}(0.1 \mathrm{~T})$ $\approx 1 / 3$. The inset shows a schematic of the pump and probe pulse configurations used in the polarization-resolved pump-probe PL experiment.

method, a circularly polarized $\left(\sigma^{+}\right.$or $\left.\sigma^{-}\right)$pump pulse creates a spin orientation of the resident electrons. ${ }^{18}$ The spin dynamics is then studied by measuring the decay of $\rho_{c}$ for the $\sigma^{+}$-polarized probe pulse delayed in time relative to the pump pulse. Pump (probe) power is kept at $1(0.05) \mathrm{mW}$, for which $B_{N} \approx 0$ [Fig. 3 (inset)]. Also, with the probe power being 20 times smaller than the pump power, it does not destroy the pump-induced spin polarization. A schematic of the pump and probe pulse configurations is shown in the inset of Fig. 4. Exploiting this method, we study the spin dynamics in a wide time range from picoseconds to milliseconds. ${ }^{5,6}$

For the study of spin dynamics in the nanosecond time regime, pump and probe pulses are derived from a picosecond Ti:sapphire laser and the pump-probe delay $\tau$ is controlled by optical delay. The polarization-selected PL originating from the probe pulse is time resolved in a streak camera to monitor the kinetics of $\rho_{c}$ for the probe-generated PL. Figure 4 shows such kinetics of $\rho_{c}$ at $\tau=2 \mathrm{~ns}$ for $\sigma^{+}$-polarized probe pulse when the pump pulse is cocircularly $\left(\sigma^{+}\right)$or cross-circularly $\left(\sigma^{-}\right)$polarized. Data are taken for $B_{\text {ext }}=0$ and $0.1 \mathrm{~T}$. As seen in Fig. 4, at times beyond $300 \mathrm{ps}$, the kinetics of $\rho_{c}$ reaches a constant value (refer to as $A_{\mathrm{CP}}$ in Fig. 1), which is strongly negative (positive) for the co- (cross-) circularly polarized pump-probe excitation. The difference $\Delta A_{\mathrm{CP}}$ between the $A_{\mathrm{CP}}$ for the two cases (co- and cross-circularly polarized pump-probe configurations) can be used as a measure of the pump-induced spin polarization of 


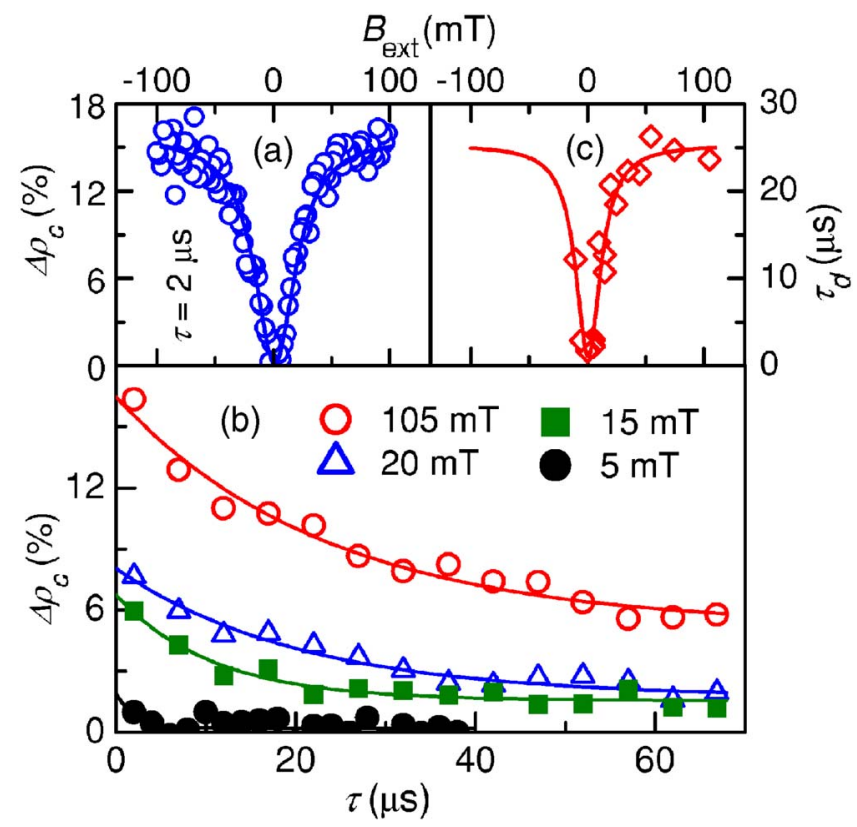

FIG. 5. (Color online) (a) Dependence of $\Delta \rho_{c}$ on $B_{\text {ext }}$ for $\tau=2 \mu$ s. The solid line is a Lorentzian fit. Total depolarization $\left(\Delta \rho_{c} \approx 0\right)$ of the electron spin is seen at $B_{\text {ext }}=0$. (b) Dependence of $\Delta \rho_{c}$ on $\tau$ at a few values of $B_{\text {ext }}$. Solid lines are exponential fits characterized by the spin decay time $\tau_{d}$, which is plotted in (c) as a function of $B_{\text {ext }}$. The solid line in (c) is a Lorentzian fit.

the resident electrons. We find that for a given $\tau, \Delta A_{\mathrm{CP}}$ is sensitive to $B_{\text {ext }}$. The difference $\Delta A_{\mathrm{CP}}(0 \mathrm{~T})$ measured at $B_{\text {ext }}=0 \mathrm{~T}$ is approximately one-third of $\Delta A_{\mathrm{CP}}(0.1 \mathrm{~T})$ measured at $B_{\text {ext }}=0.1 \mathrm{~T}$ for $\tau=2 \mathrm{~ns}$ (Fig. 4). An identical behavior is seen when measured at $\tau=10$ ns. Following the theory of Merkulov et al., ${ }^{10}$ we conclude that for $B_{\text {ext }}=0$, a partial relaxation (up to one-third of the initial value) of electron-spin polarization due to the FFNS takes place within a time shorter than 2 ns. However, the remaining spin polarization does not decay up to $10 \mathrm{~ns}$. In the presence of $B_{\text {ext }}=0.1 \mathrm{~T}$, electron-spin relaxation by the FFNS is suppressed. In that case, electron-spin relaxation time is much longer than $10 \mathrm{~ns}$.

As discussed in Sec. I, for $B_{\text {ext }}=0$, decay of the electronspin polarization surviving after $2 \mathrm{~ns}$ (one-third of the initial value) is governed by the NSPE over a longer time scale. ${ }^{10}$ For the study of spin dynamics in the microsecond time scale, it is more convenient to measure the magnetic-field and delay dependences of $\rho_{c}$ integrated over the PL lifetime. In these experiments, the pump and probe pulses are derived from a cw Ti:sapphire laser by using acousto-optic modulators (AOMs), which act as electrically controlled gates. Pump and probe pulse widths $(1 \mu$ s each) and the delay $\tau$ between them are controlled by sending electrical pulses to the AOMs from a programmable function generator. In this case, the accessible range of $\tau$ is not limited by the laser pulse repetition period of $12 \mathrm{~ns}$. Details of the experimental setup are discussed in Ref. 6. We measure the difference $\Delta \rho_{c}$ between $\rho_{c}$ (integrated over the PL lifetime) for the probe PL for cross- and cocircularly polarized pump-probe excitations.

At first, we measure the dependence of $\Delta \rho_{c}$ on $B_{\text {ext }}$ at $\tau=2 \mu$ s [Fig. 5(a)]. Near $B_{\text {ext }}=0$, we find that $\Delta \rho_{c} \approx 0$. This suggests that for $B_{\text {ext }}=0$, a total depolarization of electron spins takes place within $2 \mu \mathrm{s}$. This is caused by the NSPE, indicating that the Larmor precession period $T_{N}$ of nuclear spins in the hyperfine field of electrons is shorter than $2 \mu \mathrm{s}$.

To obtain a more quantitative estimate of $T_{N}$, we perform a systematic measurement of electron-spin polarization decay time $\tau_{d}$ as a function of $B_{\text {ext }}$. Figure 5(b) shows the delay dependence of $\Delta \rho_{c}$ at different values of $B_{\text {ext }}$. The decay of $\Delta \rho_{c}$ with $\tau$ can be well approximated by an exponential function, $\Delta \rho_{c}=A_{0}+A_{1} \exp \left(-\tau / \tau_{d}\right)\left[\right.$ Fig. 5(b)]. The decay time $\tau_{d}$ obtained from such fits is shown in Fig. 5(c) for different values of $B_{\text {ext }}$. It is seen that $\tau_{d}$ decreases down to $1 \mu$ s when $\left|B_{\text {ext }}\right|$ goes to zero, for which electron-spin relaxation takes place due to hyperfine interaction with nuclei. ${ }^{35}$ At low temperatures, the total depolarization of the electron spins under the vanishing external magnetic field is caused by the NSPE in the microsecond time scale. ${ }^{10}$ Therefore, we assign the time $1 \mu$ s obtained from Fig. 5(c) at $B_{\text {ext }}=0$ as an upper limit of $T_{N}{ }^{36}$ No other experimental study of $T_{N}$ in QDs is available, to our knowledge, in the literature for a comparison with our estimate of $T_{N}$. However, the value of $T_{N} \sim 1 \mu \mathrm{s}$ obtained by us for the self-assembled InP QDs is comparable to the theoretical estimate of $T_{N}$ for GaAs QDs. ${ }^{10}$ This agreement suggests that $T_{N}$ may have the same order of magnitude $(\sim 1 \mu \mathrm{s})$ in different self-assembled III-V quantum dots.

\section{CONCLUSION}

The effects of nuclear spins on the electron-spin dynamics in singly negatively charged InP QDs are studied at low temperature $(T \approx 5 \mathrm{~K})$, where hyperfine interaction with the nuclear spins is the dominant relaxation channel for the electron spins. We observe that at the vanishing external magnetic field, partial relaxation (up to one-third of the initial value) of electron-spin polarization takes place within $\sim 1 \mathrm{~ns}$ due to the frozen fluctuations of the nuclear hyperfine field. A value of $15 \mathrm{mT}$ is estimated for the effective magnetic field $B_{f}$ of the frozen fluctuations of nuclear spins. Total depolarization of electron spins with a characteristic time of $1 \mu \mathrm{s}$ is observed at the vanishing external magnetic field, due to the slow variation of $B_{f}$ in time caused by the nuclearspin precession in the hyperfine field of electrons. The characteristic time of $1 \mu \mathrm{s}$ is assigned to the nuclear-spin precession period $T_{N}$ in the hyperfine field of electrons. At high excitation power, the dynamic nuclear polarization is observed, giving rise to an Overhauser field $B_{N}=6 \mathrm{mT}$ at $50 \mathrm{~mW}$ excitation.

\section{ACKNOWLEDGMENTS}

The authors thank V. K. Kalevich and I. Ya. Gerlovin for fruitful discussions. This work is partially supported by "Grants-in-Aid for Scientific Research" No. 17-5056, No. 13852003, and No. 18204028 from the MEXT of Japan and "R\&D Promotion Scheme Funding International Joint Research" promoted by NICT of Japan, by ISTC Grant No. 2679, by the Russian Ministry of Science and Education Grant No. RNP.2.1.1.362, and by the Russian Fund for Basic Research Grant No. 06-02-17137-a. 
*Email address: bipulpal@ sakura.cc.tsukuba.ac.jp

†Email address: syuv54@mail.ru

${ }^{1}$ D. Gammon, Al. L. Efros, T. A. Kennedy, M. Rosen, D. S. Katzer, D. Park, S. W. Brown, V. L. Korenev, and I. A. Merkulov, Phys. Rev. Lett. 86, 5176 (2001).

${ }^{2}$ Optical Orientation, edited by F. Meier and B. P. Zakharchenya, Modern Problems in Condensed Matter Sciences Vol. 8 (NorthHolland, Amsterdam, 1984).

${ }^{3}$ R. I. Dzhioev, B. P. Zakharchenya, V. L. Korenev, P. E. Pak, M. N. Tkachuk, D. A. Vinokurov, and I. S. Tarasov, Pis'ma Zh. Eksp. Teor. Fiz. 68, 711 (1998) [JETP Lett. 68, 745 (1998)].

${ }^{4}$ R. I. Dzhioev, B. P. Zakharchenya, V. L. Korenev, and M. V. Lazarev, Fiz. Tverd. Tela (S.-Peterburg) 41, 2193 (1999) [Phys. Solid State 41, 2014 (1999)].

${ }^{5}$ M. Ikezawa, B. Pal, Y. Masumoto, I. V. Ignatiev, S. Yu. Verbin, and I. Ya. Gerlovin, Phys. Rev. B 72, 153302 (2005).

${ }^{6}$ B. Pal, M. Ikezawa, Y. Masumoto, and I. V. Ignatiev, J. Phys. Soc. Jpn. 75, 054702 (2006).

${ }^{7}$ Semiconductor Spintronics and Quantum Computation, edited by D. D. Awschalom, D. Loss, and N. Samarth, Nanoscience and Technology (Springer, Berlin, 2002).

${ }^{8}$ D. Loss and D. P. DiVincenzo, Phys. Rev. A 57, 120 (1998).

${ }^{9}$ V. L. Berkovits, A. I. Ekimov, and V. I. Safarov, Zh. Eksp. Teor. Fiz. 65, 346 (1973) [Sov. Phys. JETP 38, 169 (1974)].

${ }^{10}$ I. A. Merkulov, Al. L. Efros, and M. Rosen, Phys. Rev. B 65 , 205309 (2002).

${ }^{11}$ A. V. Khaetskii, D. Loss, and L. Glazman, Phys. Rev. Lett. 88, 186802 (2002).

${ }^{12}$ Y. G. Semenov and K. W. Kim, Phys. Rev. B 67, 073301 (2003).

${ }^{13}$ P.-F. Braun, X. Marie, L. Lombez, B. Urbaszek, T. Amand, P. Renucci, V. K. Kalevich, K. V. Kavokin, O. Krebs, P. Voisin, and Y. Masumoto, Phys. Rev. Lett. 94, 116601 (2005).

${ }^{14}$ See, e.g., Fig. 3 of Ref. 13.

${ }^{15}$ S. Cortez, O. Krebs, S. Laurent, M. Senes, X. Marie, P. Voisin, R. Ferreira, G. Bastard, J.-M. Gérard, and T. Amand, Phys. Rev. Lett. 89, 207401 (2002).

${ }^{16}$ J. S. Colton, T. A. Kennedy, A. S. Bracker, and D. Gammon, Phys. Rev. B 69, 121307(R) (2004).

${ }^{17}$ I. E. Kozin, V. G. Davydov, I. V. Ignatiev, A. V. Kavokin, K. V. Kavokin, G. Malpuech, H.-W. Ren, M. Sugisaki, S. Sugou, and Y. Masumoto, Phys. Rev. B 65, 241312(R) (2002).

${ }^{18} \mathrm{~A}$ model of optical orientation of resident electron spins for quasiresonant excitation in the excited state of a singly negatively charged QDs, applicable for our experimental condition, was discussed in detail in Ref. 6.

${ }^{19}$ Y. Masumoto, S. Oguchi, B. Pal, and M. Ikezawa, Phys. Rev. B 74, 205332 (2006).

${ }^{20}$ Recently, negative degree of circular polarization of PL from the negatively charged QDs has been reported by other researchers, see, e.g., Refs. 15 and 21-23. Unlike the quasiresonant excitation in the QD excited state used in our experiments, measure- ments of Refs. 15 and 21-23 have been performed under wetting layer excitation and the holes were assumed to be totally depolarized before capturing in the QDs.

${ }^{21}$ S. Laurent, O. Krebs, S. Cortez, M. Sénès, X. Marie, T. Amand, P. Voisin, and J.-M. Gérard, Physica E (Amsterdam) 20, 404 (2004).

${ }^{22}$ V. K. Kalevich, I. A. Merkulov, A. Y. Shiryaev, K. V. Kavokin, M. Ikezawa, T. Okuno, P. N. Brunkov, A. E. Zhukov, V. M. Ustinov, and Y. Masumoto, Phys. Rev. B 72, 045325 (2005).

${ }^{23}$ S. Laurent, M. Sénès, O. Krebs, V. K. Kalevich, B. Urbaszek, X. Marie, T. Amand, and P. Voisin, Phys. Rev. B 73, 235302 (2006).

${ }^{24}$ This behavior is similar to that observed for optical pumping of electron spins in $n$-type semiconductors (Ref. 2). We estimate that the number of photocreated electron-hole pairs per QD per pulse remains less than 1 up to the highest average excitation power $(50 \mathrm{~mW})$ used.

${ }^{25}$ M. E. Ware, E. A. Stinaff, D. Gammon, M. F. Doty, A. S. Bracker, D. Gershoni, V. L. Korenev, S. C. Badescu, Y. LyandaGeller, and T. L. Reinecke, Phys. Rev. Lett. 95, 177403 (2005).

${ }^{26}$ K. V. Kavokin, Phys. Status Solidi A 195, 592 (2003).

${ }^{27}$ T. Flissikowski, I. A. Akimov, A. Hundt, and F. Henneberger, Phys. Rev. B 68, 161309(R) (2001).

${ }^{28}$ S. Laurent, B. Eble, O. Krebs, A. Lemaître, B. Urbaszek, X. Marie, T. Amand, and P. Voisin, Phys. Rev. Lett. 94, 147401 (2005).

${ }^{29}$ Y. Masumoto, I. V. Ignatiev, K. Nishibayashi, T. Okuno, S. Yu. Verbin, and I. A. Yugova, J. Lumin. 108, 177 (2004).

${ }^{30}$ The minimum of $\left|\rho_{c}\right|$, appears at $B_{\text {ext }} \approx 0$, when the polarization of the excitation beam is modulated between $\sigma^{+}$, and $\sigma^{-}$, at a frequency of $42 \mathrm{kHz}$, by using a photoelastic modulator in the excitation path. It is well known that in such a case, dynamic nuclear polarization cannot build up (Ref. 2).

${ }^{31}$ S. W. Brown, T. A. Kennedy, D. Gammon, and E. S. Snow, Phys. Rev. B 54, R17339 (1996).

${ }^{32}$ T. Yokoi, S. Adachi, H. Sasakura, S. Muto, H. Z. Song, T. Usuki, and S. Hirose, Phys. Rev. B 71, 041307(R) (2005).

${ }^{33}$ A. Imamoglu, E. Knill, L. Tian, and P. Zoller, Phys. Rev. Lett. 91, $017402(2003)$

${ }^{34}$ G. Salis, D. D. Awschalom, Y. Ohno, and H. Ohno, Phys. Rev. B 64, 195304 (2001).

${ }^{35}$ For $B_{\text {ext }} \neq 0$, the effect of hyperfine interaction on the electronspin relaxation begins to diminish, because the external magnetic field starts to influence the electron-nuclear spin dynamics. Electron-spin decay time $\tau_{d}$, measured for $\left|B_{\text {ext }}\right|>0$, cannot be directly assigned to the nuclear spin precession period $T_{N}$, in the hyperfine field of electrons. Effects of the external magnetic field on the electron-nuclear spin dynamics are discussed in Ref. 10.

${ }^{36}$ Accuracy of this estimate is decided by the accuracy of the exponential fit, estimated at $30 \%$ in Fig. 5(b). 